\title{
Object Identification Using Bar Codes Based on LADAR Intensity ${ }^{1}$
}

\author{
William C. Stone ${ }^{2}$, Geraldine S. Cheok ${ }^{2}$, Karen M. Furlani ${ }^{2}$, David Gilsinn ${ }^{3}$ \\ National Institute of Standards and Technology \\ Gaithersburg, MD 20899, USA
}

\begin{abstract}
Tests of a novel concept for tracking construction components were carried out at the National Institute of Standards and Technology. The approach makes use of laser radar and retro-reflective barcodes attached to manufactured building materials. Tests were conducted for various barcode sizes and spacings through a variety of ranges to a maximum of $40 \mathrm{~m}$. At $40 \mathrm{~m}$, it was possible to infer one byte of information content in a 1D barcode measuring $575 \mathrm{~mm}$ (22.6 in.) in length with no special optical processing for a LADAR instrument with an aperture beam diameter of $25 \mathrm{~mm}$ and a beam divergence of $2.7 \mathrm{mrad}$. Simple thresholding post-processing techniques were employed to study the resulting intensity data and to deduce minimum detectable bar gap spacing. These analyses have suggested paths to significant resolution enhancements based on image processing and optical physics simulation techniques.
\end{abstract}

Keywords: LADAR, object recognition, pattern recognition, construction automation, bar codes, intensity, image processing.

\section{INTRODUCTION}

Imaging sensors such as LADARs (laser distance and ranging devices) are used to rapidly acquire data of a scene to generate $3 \mathrm{D}$ models. The increased interest in this technology is due to the substantial growth in applications for real-time scene updates driven by the recent advances in imaging sensor software and hardware. Current applications include site surveillance, map/terrain update/reconnaissance, indoor/outdoor visual inspection, automatic navigation, and collision avoidance.

Imaging sensors are used to obtain two- or threedimensional arrays of values such as range, intensity, or other characteristics of a scene. Currently available LADARs can gather four pieces of information - range to an object; two spatial angular measurements; and the strength of the returned signal (intensity); some instruments provide color intensity as separate RGB channels. Various methods are used to convert the data, which are collected in the form of point clouds, into meaningful 3-D models of the actual environment for visualization and scene

During the past year, the National Institute of Standards and Technology (NIST) has initiated a new interpretation. The need for accurate representations varies with the purpose of the application. In the construction industry, an accurate representation aids in determining payment for completed work, determining if errors are being made, and in tracking work progress on a project. In autonomous navigation, an accurate representation would result in crash avoidance and successful course navigation. In target acquisition, an accurate model could mean the difference between hitting or missing a target.

The points within a point cloud are indistinguishable from each other with regard to their origin; i.e., there is no way to tell if a point is reflected from a tree or from a building. As a result, the methods used to generate the models treat all points identically and the results are indistinguishable "humps/bumps" in the scene. Current surface generation methods using LADAR data require intensive manual intervention to recognize, replace, and/or remove objects within a scene. As a result, aids to object identification have been recognized by the end users as a highly desirable feature and a high priority area of research.

project to explore and evaluate the concept of a hybrid LADAR - a LADAR that can simultaneously

\footnotetext{
${ }^{1}$ Official constribution of the National Institute of Standards and Technology; not subject to copyright in the United States

${ }^{2}$ Building and Fire Research Lab, Construction Metrology and Automation Group: william.stone@nist.gov, cheok@nist.gov, karen.furlani@nist.gov

${ }^{3}$ Information Technology Lab, Mathematical and Computational Sciences Division: david.gilsinn@nist.gov
} 
acquire "intelligent" information about an object in addition to existing generic point cloud data when conducting a scan of a scene such as a construction site. The combined information from this type of sensor would be a major step towards the ultimate goal of automated tracking of manufactured components on a construction site.

This paper will describe the proposed work at NIST and the initial efforts in the project.

\section{BACKGROUND}

The project's proposed work involves investigating 1 ) the ability of using a LADAR to acquire information (such as type of material, distinct patterns, fiducial point identifiers, etc.) about an object in addition to range data, and 2) the fusion of LADAR and other sensors. These "other sensors" could be a) RFID tags that contain encrypted data about the scanned object, i.e, beam, column, part number, etc., b) micro-reflective sensors arranged in a standardized pattern or at pre-determined fiducial locations on an object to allow for identification, and/or c) a camera to provide a concurrent image of a scanned scene. This paper discusses the second aspect -- that of using retroreflective barcodes in an effort to extract the identity of a component within a LADAR scan scene.

The additional information from the hybrid LADAR does not identify objects per se, but is used to aid in object identification. Existing image processing algorithms include object identification based on colors, spin image processing (SIP), and a volumetric approach using octrees. These algorithms are currently research tools that have been successfully used to identify a limited number of objects in a scene. The effectiveness and accuracy of these methods for identifying objects in a construction scene where low object-to-scene point ratios exist, has not yet been verified. Further, all have the distinct disadvantage that they must initially operate on the entire 3-D data set and therefore represent computationally expensive standalone approaches. Furthermore, there presently are no standard protocols (point density; object size, shape, and placement; partial obstruction; etc.) to measure the accuracy of these algorithms.

Some further challenges include: determining what lasers can or cannot be used for object detection; if RFID tags are used, examining increased range, optimum sizing, and power requirements; interference of sensor signal by the surrounding objects in a scene; and non-line-of-sight detection.

The project is divided into four phases. The first phase will involve investigating various technologies that can be combined with LADAR to yield additional information about a scene to aid in object identification. The second phase focuses on detailing system requirements to combine the capabilities of a LADAR with the most promising technology(s) identified in the first phase. The third phase involves testing and evaluating the LADAR and aids to object identification in the construction environment. The fourth phase will be developing a standard protocol and measures of performance for the various object recognition algorithms determined to be suited for construction scene.

\section{2-D BAR CODES}

The use of bar codes or UPC (Universal Product Code) symbols has become the universal method for the rapid identification of objects ranging from produce to airplane parts. The same method could also be used to identify objects within a construction scene. This would involve using the LADAR to "read" a bar code. The concept is to use the intensity data from the LADAR to distinguish the bar pattern. The advantage of this concept is that no additional hardware or other sensor data is required to gather the additional data. The basis for this concept lies in the high intensity values obtained from highly reflective materials. Software will have to be developed to read the intensity patterns.

The challenges are the ability to read the bar code from $100 \mathrm{~m}$ or greater, distinguish bar code points from the other points in the scene, capture sufficient points to define the bar code for correct identification, and the ability to read bar codes that are skewed.

\subsection{Preliminary Resolution Tests}

A Riegl $^{4}$ scanner was used for all the experiments. The intensity values for this scanner ranged from 1 to 255 (most reflective) and the intensity is a function of the strength of the returned signal. For the initial tests, 3M's Long Distance Performance (LDP) reflective sheeting was used to construct the bar codes. This material is a highly reflective prismatic lens sheeting used for traffic signage.

\footnotetext{
${ }^{4}$ Certain trade names and company products are mentioned in the text or identified in an illustration in order to adequately specify the experimental procedure and equipment used. In no case does such an identification imply recommendation or endorsement by the National Institute of Standards and Technology, nor does it imply that the products are necessarily the best available for the purpose.
} 


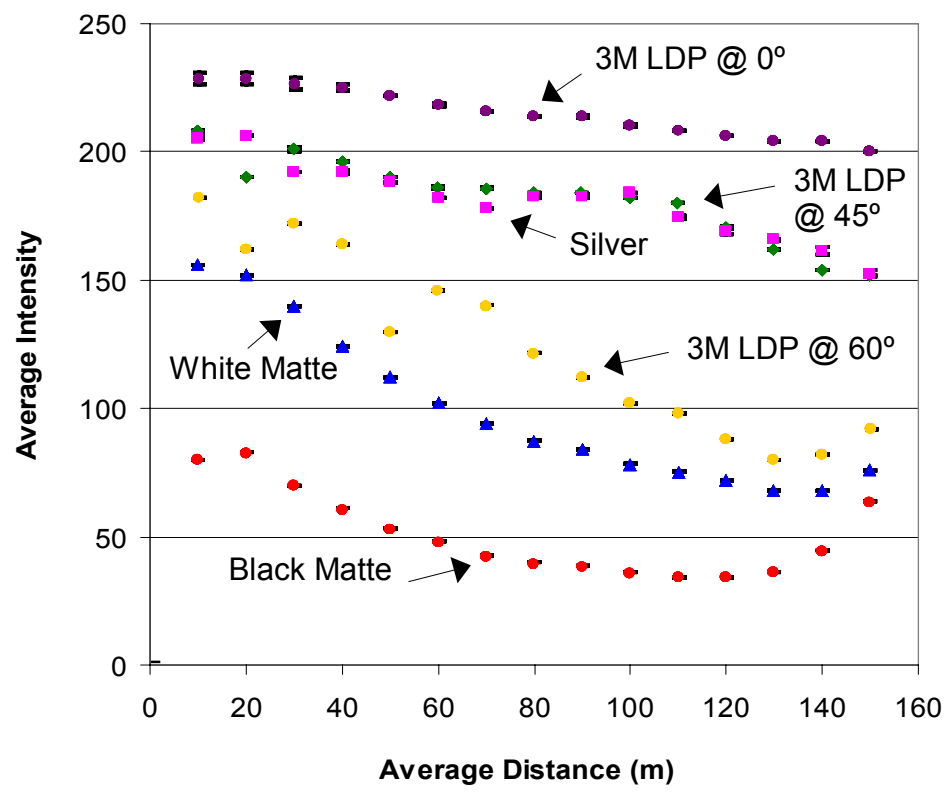

Figure 1. Intensity vs. Distance for different material color. Error bars are $95 \%$ confidence intervals.

\section{$\underline{\text { Intensity vs. Distance }}$}

The effect on the intensity value as a function of distance was examined by taking readings at $10 \mathrm{~m}$ intervals starting from $10 \mathrm{~m}$ to $150 \mathrm{~m}$. Four targets were created using aluminum sheets, $508 \mathrm{~mm}(\mathrm{H}) \mathrm{x}$ $406.4 \mathrm{~mm}$ (W) (20 in. $\mathrm{x} 16$ in.), that were: 1) painted matte black; 2) painted matte white; 3) left unpainted (shiny silver); and 4) covered with a sheet of LDP material. The LDP targets were set at three orientations: $0^{\circ}$ (perpendicular to laser beam), $45^{\circ}$, and $60^{\circ}$. At each distance, 10 readings were recorded. The plot of the average intensity vs. distance is shown in Fig. 1. As seen in Fig. 1, the intensities for the LDP material at $0^{\circ}$ are above 200 for distances up to $150 \mathrm{~m}$; thereby making these points easily distinguishable from the other points in a typical scene. However, if the targets were rotated away from the laser, these values are not as easily distinguishable - see data for $\operatorname{LDP} 45^{\circ}$ and $60^{\circ}$.

\section{$\underline{\text { Bar Patterns }}$}

Once it was determined that the reflective material produced sufficiently high intensity values to be used to construct the bar codes, the next step was to determine if a bar code pattern could be recognized with human intervention.

The first test scanned bars of varying width, set at a fixed spacing of $152.4 \mathrm{~mm}$ (3 in.) and a distance of approximately $8.7 \mathrm{~m}$. Three LDP bars were attached to a wooden board: $292.1 \mathrm{~mm}(\mathrm{H}) \times 152.4 \mathrm{~mm}(\mathrm{~W})($ 11.5 in. $x 6$ in.), $291.1 \mathrm{~mm} \mathrm{x} 76.2 \mathrm{~mm}$ (11.5 in. $\mathrm{x} 3$ in.), and $291.1 \mathrm{~mm} \mathrm{x} 38.1 \mathrm{~mm}$ ( 11.5 in. $\times 1.5$ in.).

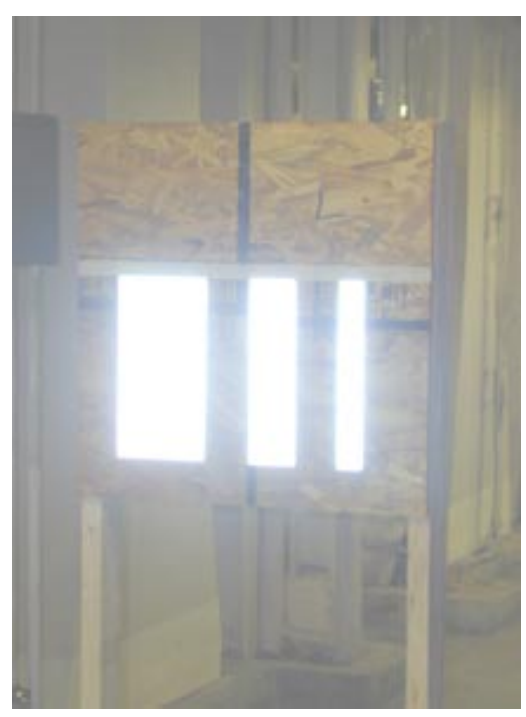

Figure 2a: Digital photograph of macro barcode test pattern.

Figure $2 \mathrm{a}$ shows a photo of the bars and Fig. $2 \mathrm{~b}$ shows a plot of the intensity values. As seen in Fig. 2 , the bar pattern is easily recognizable.

The next step was to test the ability to distinguish bar patterns at various distances. The objective was to determine the minimum size bar and the minimum spacing between bars required to distinguish the bar patterns at various distances. A series of test scenes were developed for various patterns and scanned at varying distances.

LDP bars were attached to a white poster board $762 \mathrm{~mm}(\mathrm{H}) \times 609.6 \mathrm{~mm}(\mathrm{~W})(30$ in. $\times 24$ in.). On each board, there were three rows of bars with 76.2 $\mathrm{mm}$ (3 in.) spacing between the rows. The top row consisted of bars at $76.2 \mathrm{~mm}$ (3 in.) spacing; 50.8 $\mathrm{mm}$ ( 2 in.) spacing in the middle row; and $25.4 \mathrm{~mm}$ (1 in.) spacing in the bottom row. Three boards were used. The bars on each board were of the same size -

Board 1: $152.4 \mathrm{~mm}(\mathrm{H}) \times 101.6 \mathrm{~mm}(\mathrm{~W})$ (6 in. x 4 in.) bars;

Board 2: $152.4 \mathrm{~mm} \times 50.8 \mathrm{~mm}$ (6 in. $\times 2$ in.) bars; Board 3: $152.4 \mathrm{~mm} \times 25.4 \mathrm{~mm}$ (6 in. $\mathrm{x} 1$ in.) bars.

The original intent was to test the bar patterns at distances of $20 \mathrm{~m}, 40 \mathrm{~m}, 60 \mathrm{~m}, 80 \mathrm{~m}$, and $100 \mathrm{~m}$. However, the images obtained at $60 \mathrm{~m}$ showed that the LDP bars were not distinguishable at that distance; therefore, it was decided to scan from shorter distances of $5 \mathrm{~m}, 10 \mathrm{~m}, 15 \mathrm{~m}, 20 \mathrm{~m}, 40 \mathrm{~m}$ and $60 \mathrm{~m}$.

Three scans were obtained for each board at each distance. The preliminary results are shown in Figs. 3, 4 and 5. For each scan, the (a) figure is a digital photo of the test board; figures (b), (c), and (d) 


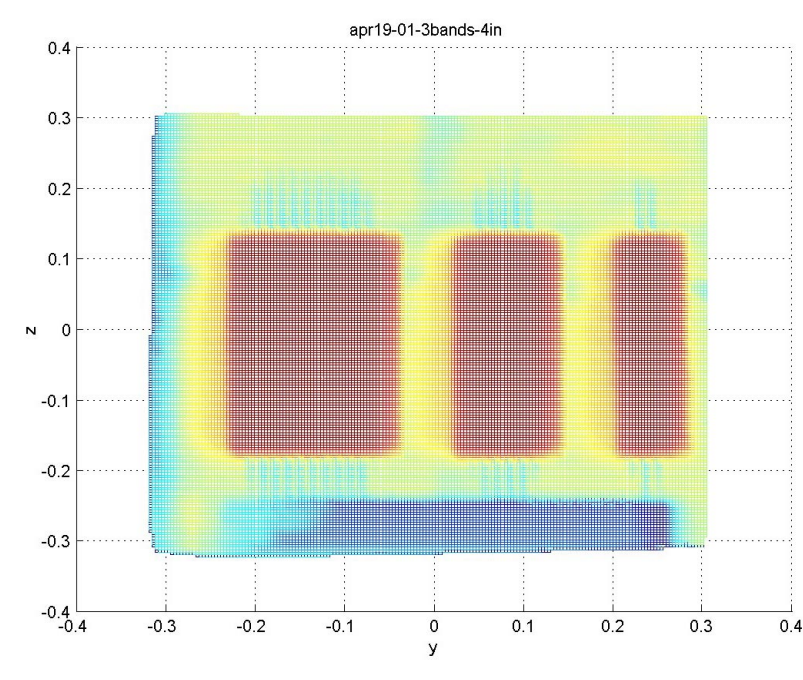

Figure 2b: 2-D plot of LADAR intensity at a range of $8.7 \mathrm{~m}$.

represent pure 2-D intensity in black and white on a scale of 0 to 255 for various test ranges. A histogram of the intensity values was evaluated to determine a threshold $[1,2]$ below which the intensity values were eliminated to reduce phantom and other unwanted points. The cropped intensity values were then plotted to provide a clear intensity image of the bar code patterns, as shown in figures (e), (f), and (g) for each of Figs. 3, 4, and 5. This method of human intervention was applied to all the data sets. Figure 6 is a histogram of a typical raw data set; the intensity values below 165 were eliminated to create the plot in Fig. 4g.

\section{DISCUSSION}

Two major observations about the three dimensional meshing technique of the intensity data used in this paper will be made. First, the figures show that at distances of $10 \mathrm{~m}$ and $20 \mathrm{~m}$ it is possible to isolate reflector bars distanced $76.2 \mathrm{~mm}$ (3 in.) and $50.8 \mathrm{~mm}$ (2 in.) apart. At a distance of $25.4 \mathrm{~mm}$ (1 in.) discrimination is problematical. A rough estimate of the separation required at $150 \mathrm{~m}$ for the current technology would then be $381 \mathrm{~mm}$ (15 in.). This is based on an extrapolation of $50.8 \mathrm{~mm}$ (2 in.) at $20 \mathrm{~m}$. A further enhancement in technology is clearly required in order to reduce this interval. The second observation is that using a histogram as a filter of the intensity response offers a significant tool to isolate the intensity response from the background as opposed to the reflectors. The current algorithm [3] uses human interaction to identify the level of intensity values to discard, but an automated algorithm might be constructed to isolate the first major peak in the histogram as frequency counts from the background. However, further exploration of this idea will be based on more experimental data.
Although the current location methodology for isolating the reflector bars depends on visualization, several techniques from 3-D image processing are currently under consideration $[4,5,6]$ as tools to automatically identify the location of the reflector bars. The first class of methods is referred to as segmentation operations. Their aim is to partition the intensity or distance data into 3-D regions that represent meaningful physical elements. The simplest segmentation method involves building a threshold (or $0 / 1$ ) function. In this method, if, for example, the intensity values that form an object occupy a certain range, then these values can be isolated by assigning them the value 1 and the background 0 . The problem with using this technique involves selecting the threshold range. Two other segmentation methods, called region growing and region splitting, aim to isolate the desired intensity or distance regions by building them up from initial estimates of their location or by continually partitioning the image to identify various homogeneous regions that can subsequently be classified [3]. Another class of methods involves examining the frequency space response of the intensity or distance data. It is known from image processing that the frequency domain representation of an image provides useful information and therefore is a candidate technique for future study.

A simplified analysis was performed on the information storage content of the data presented herein. At a distance of $40 \mathrm{~m}$ it was concluded that a "macro" 1-D barcode that conveyed one byte ( 8 bits) of information would have to be $575 \mathrm{~mm}$ (22.6 in.) in length. We believe that at least an order of magnitude improvement can be achieved through the use of image processing techniques and 2-D barcodes using the same approach.

\section{CONCLUSIONS}

The authors have investigated an approach to resolving the problem of tracking large numbers of components present at a typical construction site. The procedure involves adding information content to the scan scene that can be subsequently postprocessed in the intensity domain of a typical LADAR data set. The intent of this approach is to provide a part ID and an associated range and bearing to the component relative to the scanner 


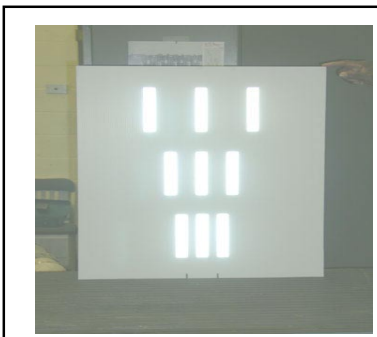

(3a) Photo

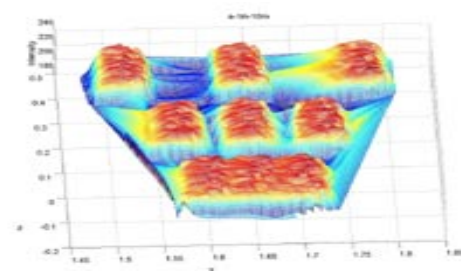

(3e) $10 \mathrm{~m}$

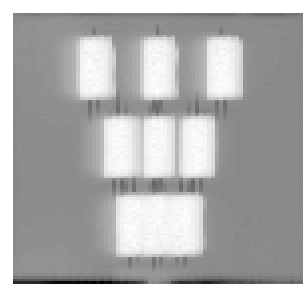

(3b) $10 \mathrm{~m}$

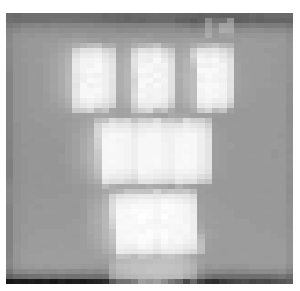

(3c) $20 \mathrm{~m}$

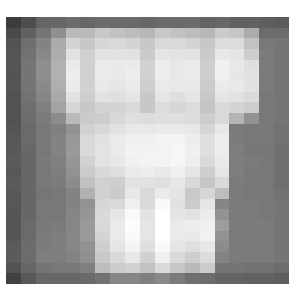

(3d) $40 \mathrm{~m}$

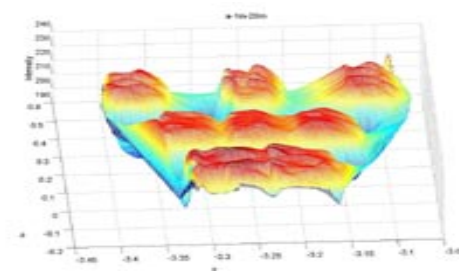

(3f) $20 \mathrm{~m}$

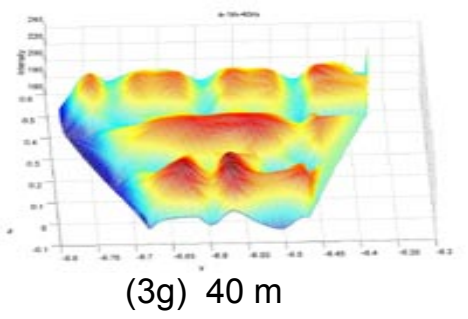

(3g) $40 \mathrm{~m}$

Figure 3: $152.4 \mathrm{~mm}$ x $25.4 \mathrm{~mm}$ (6 in. $\mathrm{x} 1$ in.) bars at varying distances.

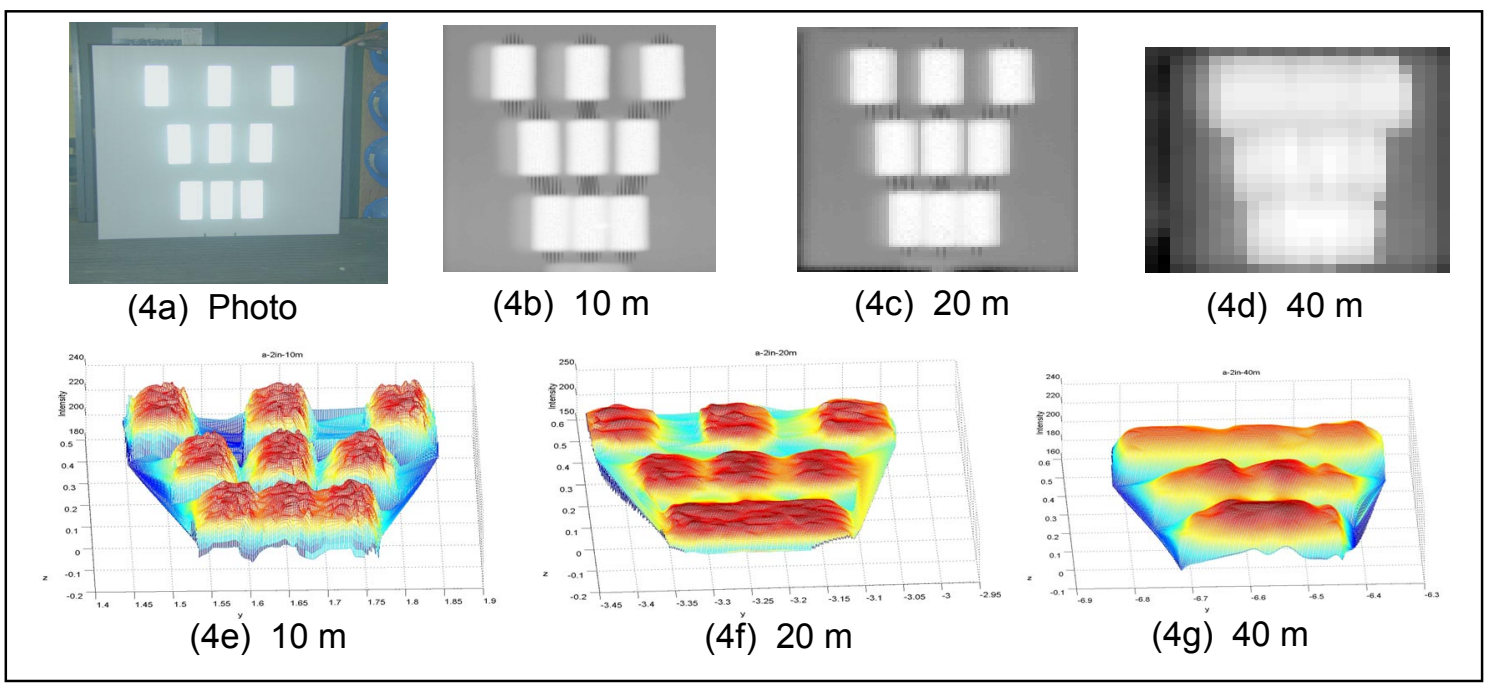

Figure 4. $152.4 \mathrm{~mm} \times 50.8 \mathrm{~mm}$ (6 in. $\times 2$ in.) bars at varying distances.

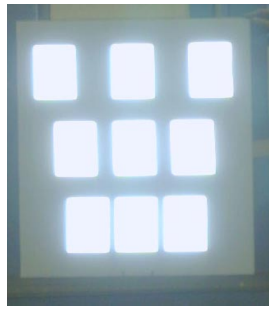

(5a) Photo

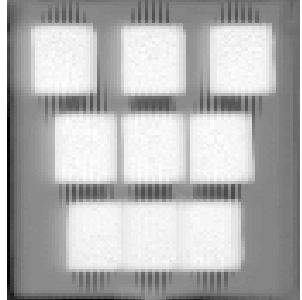

(5b) $10 \mathrm{~m}$

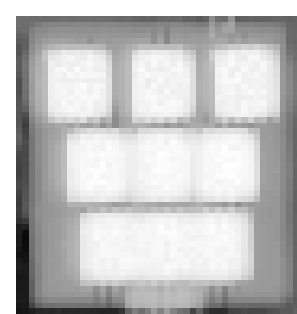

(5c) $20 \mathrm{~m}$

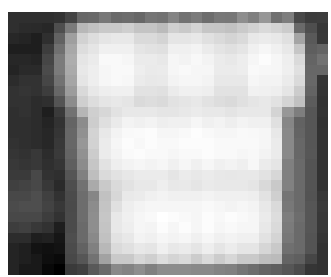

(5d) $40 \mathrm{~m}$

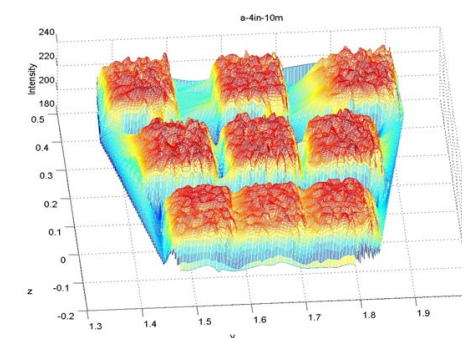

(5e) $10 \mathrm{~m}$

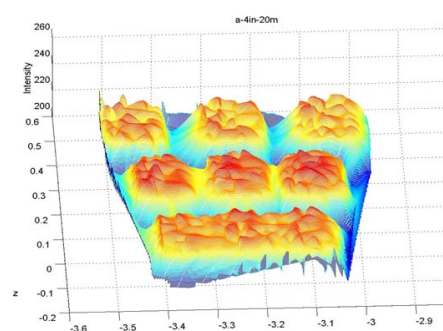

(5f) $20 \mathrm{~m}$

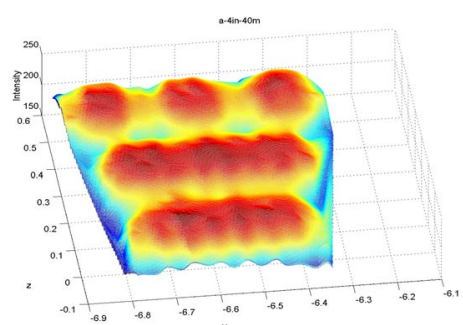

(5g) $40 \mathrm{~m}$

Figure 5. $152.4 \mathrm{~mm} \times 101.6 \mathrm{~mm}$ (6 in. $\mathrm{x} 4$ in.) bars at varying distances. 


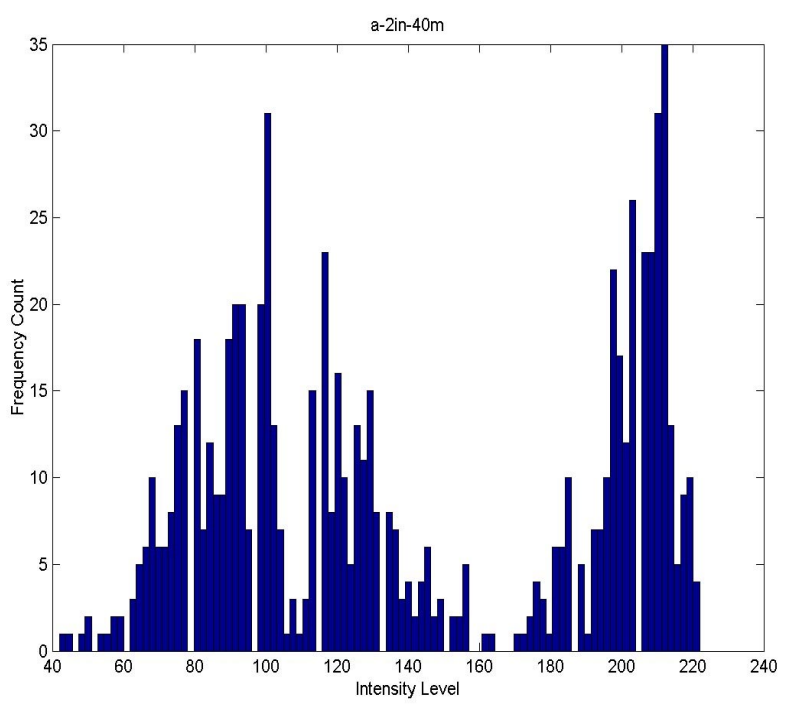

Figure 6. Histogram of Intensity Levels for 152.4 $\mathrm{mm} \times 50.8 \mathrm{~mm}$ (6 in. $\times 2$ in.) bars at $40 \mathrm{~m}$.

position. This will permit a much more rapid determination of the part's position and orientation using present object recognition techniques, since they can now operate on a drastically reduced data set.

\section{REFERENCES}

1. William K. Pratt, Digital Image Processing, John Wiley \& Sons, New York, 1991, pp. 534541.

2. Dwayne Phillips, Image Processing in $C, \mathrm{R} \& \mathrm{D}$ Publications, Inc., Lawrence, Kansas, 1994, pp. 345-396.

3. N. Nikolaidis, I. Pitas, 3-D Image Processing Algorithms, John Wiley \& Sons, Inc., New York, 2001.

4. J., -A. Beraldin, S. F. El-Hakim, F. Blais, "Performance Evaluation of Three Active Vision Systems Built at the National Research Council of Canada", Proc. Conference on Optical 3-D Measurement Techniques, Vienna, Austria, Oct. 2-4, 1995, 352-362.

5. Ernest L. Hall, Computer Image Processing and Recognition, Academic Press, New York, 1979, pp. 370-412.

6. J. -A. Beraldin, L. Cournoyer, M. Rioux, S. F. El-Hakim, G. Godin, "Object model creation from multiple range images: Acquisition, Calibration, Model Building and Verification", Proc. Int. Conf. on Recent Advances in 3-D Digital Imaging and Modeling, Ottawa, Ont., May 12-15, 1997, 326-333. 